\title{
APPROXIMATE THEORETICAL SOLUTIONS FOR FLOATING AN SUBMERGED BREAKWATERS
}

heikal e.m

\begin{abstract}
The simple two solutions for determining the efficiency of vertical floating and submerged breakwaters are analyzing it by using the Eigenfunction Expansion Method. These two solutions carried out for the propagating wave mode only in two dimensions. The transmission and reflection coefficients were calculated for different structure and wave conditions. Also, the wave height distribution around the breakwater was calculated. The validity of these models is obtained by comparing the results with the obtained results from both the exact solutions and the available experimental data. It is concluded that; the approximate solutions give good agreement with the exact solutions and the experimental data especially for the small dimensionless wave number.
\end{abstract}

